\title{
Do pictures of faces, and which ones, capture attention in the inattentional-blindness paradigm?
}

Christel Devue, Cédric Laloyaux, Dorothée Feyers, Jan Theeuwes $\uparrow$, Serge Brédart

Cognitive Science Department, University of Liège, 5 Boulevard du Rectorat (Bât. B32), 4000 Liège, Belgium; Cognitive Psychology, Vrije Universiteit Amsterdam, Amsterdam, The Netherlands Received 28 February 2008, in revised form 21 August 2008; published online 17 April 2009

\begin{abstract}
Faces and self-referential material (eg one's own name) are more likely to capture attention in the inattentional-blindness (IB) paradigm than other stimuli. This effect is presumably due to the meaning of these stimuli rather than to their familiarity [Mack and Rock, 1998 Inattentional Blindness (Cambridge, MA: MIT Press)]. In previous work, IB has been investigated mostly with schematic stimuli. In the present study, the generalisability of this finding was tested with photographic stimuli. In support of the view that faces constitute a special category of stimuli, pictures of faces were found to resist more to IB than pictures of common objects (experiment 1) or than pictures of inverted faces (experiment 2). In a third experiment, the influence of face familiarity and identity (the participant's own face, a friend's face, and an unknown face) on IB rates was evaluated. Unexpectedly, no differential resistence to blindness across these three kinds of faces was found. In conclusion, pictures of faces attracted attention more than pictures of objects or inverted faces in the IB paradigm. However, this effect was not dependent on face familiarity or identity.
\end{abstract}

\section{Introduction}

Inattentional blindness (IB) occurs when an observer fails to detect the unexpected presence of a stimulus in his/her visual field. This phenomenon was discovered by Mack and Rock (1998) with a paradigm they designed to investigate the relationships between perception and attention. In this paradigm, observers focus on a length-judgment task that consists in reporting which arm (vertical or horizontal) of a flashed large cross is longer than the other. This procedure is used in two or three trials (non-critical trials). Then, in the third or fourth trial (the critical trial), a critical stimulus (CS) is unexpectedly presented beside the large cross (see figure 1). At this instant, observers are in the condition of inattention since they could not expect the appearance of the CS. Immediately after the length-judgment task, they are questioned about their detection of this CS and are asked to describe it or to pick it out from a set of alternatives. After two or three non-critical trials, the CS is presented a second time. Observers are then in the condition of divided attention, since they can expect the appearance of a CS.

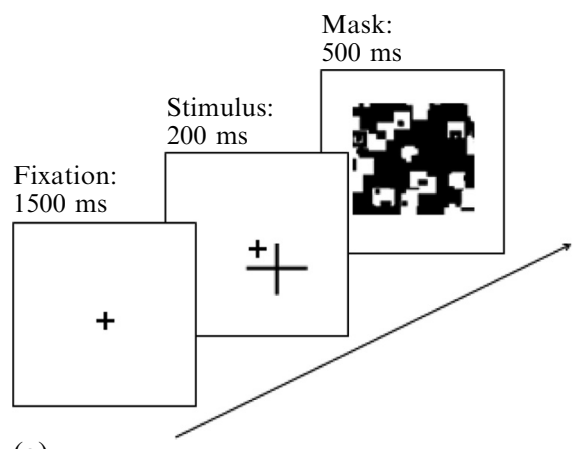

(a)

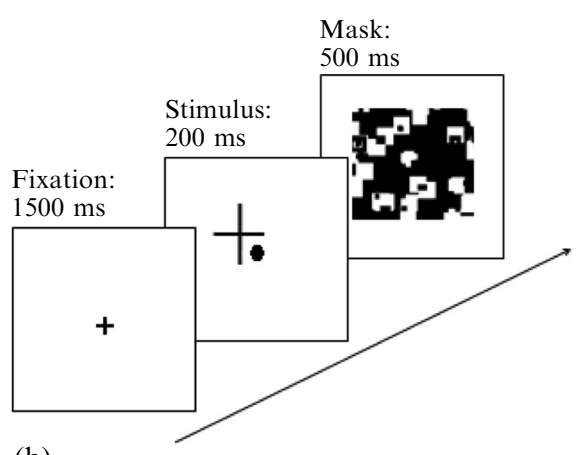

(b)

Figure 1. Presentation sequence of a non-critical trial (a) and critical trial (b): the critical stimulus (CS) appears at fixation beside the large cross (adapted from Mack and Rock 1998). 
Finally, in a last control trial, observers are instructed to ignore the length-judgment task and to stare at fixation. Full attention is thus devoted to the processing of the CS allowing the verification of its perceptibility.

In Mack and Rock's early experiments, CS consisted of simple geometric shapes (eg a square, a coloured spot). They found that a large proportion of observers (up to $85 \%$ ) failed to detect such CSs and concluded that perception requires attention or, in other words, that attention must first be captured before perception can occur. Then, the possibility of certain important and meaningful stimuli being particularly prone to capture attention was examined.

Mack and Rock (1998) used their participants' own names as CSs to address this question. Indeed, own name has been described as particularly important with regard to its capacity to grab attention (eg by comparison with neutral words-Moray 1959). Accordingly, Mack and Rock found that one's own name was more resistant to IB than other stimuli (eg other names or very frequent words). Hence, they concluded that one's own name captures attention because of its importance (rather than because of its lexicality or familiarity) which is in favour of a late-selection theory (Deutsch and Deutsch 1963) which assumes that attentional selection occurs at a relatively 'late' stage of visual processing.

Mack and Rock (1998) likewise tested whether faces (another important stimulussee eg Bruce 1998) would be better detected than other objects. They used cartoon-like faces and found that a happy-face icon was more resistant to IB than other kinds of CS (sad, neutral, scrambled, or inverted happy faces, as well as circles). Since they also found that IB rates were significantly lower when one's own name or the happy-face icon were presented than when a very frequent word (eg 'the') was presented under comparable conditions, they concluded that familiarity alone cannot account for the detection of one's own name or the happy-face icon. Later, Mack et al (2002) confirmed, using three different paradigms (attentional blink, RSVP with varying load, and stimulus crowding), the notion that one's own name and a happy-face icon capture attention because of their importance.

Studies on patients also demonstrated the influence of such important stimuli on attention. For instance, Vuilleumier and Schwartz (2001) showed that faces and emotional expressions can be processed despite lying on the unattended hemifield in brain-damaged patients presenting hemi-neglect. Recently, Perrin et al (2006), using ERP, showed that minimally conscious patients and some vegetative-state patients present a differential P3 component in response to their own name by comparison with other names.

In sum, these studies thus suggest that stimuli of great social importance have the ability to capture attention. However, faces used in Mack and Rock's (1998) experiments were cartoon-like happy faces and it is unknown whether the effects would still hold if more realistic stimuli had been presented. Other studies with photographic stimuli in change detection paradigms have indicated that faces seem to be prioritised in terms of allocation of attention. For instance, David et al (2006) showed that, in natural scenes, gradual changes in facial expressions were better detected than gradual changes in objects' colour. Similarly, Ro et al (2001) showed that changes to a single face presented among objects of different categories were better detected than changes to these objects (but see Palermo and Rhodes 2003). These studies thus suggest that realistic pictures of faces are preferentially attended to as compared to pictures of other objects, but they do not demonstrate that faces capture attention. Recently, however, Theeuwes and Van der Stigchel (2006) used the inhibition-of-return phenomenon to show that, when a face and an object are simultaneously presented, attention automatically shifts towards the location of the face. Hence, a realistic facial stimulus might also capture attention in the IB paradigm. 
The first aim of this study was to assess this hypothesis. In experiment 1 , we tested whether pictures of faces resist more to IB than pictures of other non-facial objects (fruits and vegetables). In addition, in experiment 2, we compared the capacity of upright faces and inverted faces (ie stimuli matched for their low-level properties) to capture attention.

The second aim of this study was to examine whether the familiarity or the identity of a face can affect its resistance to IB. More specifically, we assessed whether one's own face that combines two properties likely to capture attention (it is both a facial and a self-relevant stimulus) is particularly resistant to IB. Indeed, the capacity of familiar and/or self-referential stimuli to capture attention remains unclear. In a change-detection task involving highly familiar (famous) and recently learned faces, Buttle and Raymond (2003) showed that changes involving a famous face were better detected than changes involving a less-familiar face. This study suggests that familiar faces could be better detected than unfamiliar ones but does not allow any predictions about one's own face. Actually, few studies (Brédart et al 2006; Devue and Brédart 2008; Laarni et al 2000; Ninomiya et al 1998; Tong and Nakayama 1999) have used own faces to investigate the attentional properties of self-referential stimuli. Previous studies used own names rather than own faces (see eg Bundesen et al 1997; Gronau et al 2003; Harris et al 2004; Harris and Pashler 2004; Kawahara and Yamada 2004; Mack et al 2002; Mack and Rock 1998; Wolford and Morrison 1980). Yet, one's own face is more exclusively a self-referential stimulus than one's own name which can be shared with other people. Moreover, control of these self-referential stimuli was sometimes inappropriate (neutral or unfamiliar stimuli were used).

In experiment 3, we therefore compared the performance obtained with one's own face, with that obtained with a highly and personally familiar face (a friend's face) or with an unknown face presented as CS. Thus, the comparison of one's own face with a friend's face would give the best possible approximation of the role of the self-referential component in attentional capture, whereas a comparison with an unknown face would inform us about familiarity effects. To assess possible bias of the participants, we incorporated a trial in which no CS was presented but which was nonetheless followed by an assessment of the detection and recognition of an additional item. This way we could determine the presence of false positives, and, more importantly, in the third experiment, whether participants were biased to see their own faces. To keep all three experiments comparable, we also added this catch trial in experiments 1 and 2.

\section{Experiment 1}

\subsection{Method}

2.1.1 Participants. Forty-eight participants (eighteen men) from the University of Liège took part in the experiment on a voluntary basis. They were naive as to the purpose of the experiment and had normal or corrected-to-normal vision. They gave their informed consent prior to their inclusion in the experiment. As the crucial point in this paradigm is that a given participant can only be subjected once to a critical trial in condition of inattention, we used a between-subjects design. Thus, participants were randomly assigned to one of two experimental conditions: 'face' $(n=24)$ or 'object' $(n=24)$ as CS.

2.1.2 Stimuli. There were eight possible CSs: four oval-shaped objects (a lemon, a strawberry, a potato, and a pear) and four unfamiliar faces (two males and two females). The size of these stimuli was about $0.9 \mathrm{deg}$ in width and $1.3 \mathrm{deg}$ in height (at a viewing distance of $56 \mathrm{~cm}$ and with a resolution of $0.035 \mathrm{~cm}$ per pixel). Each facial stimulus was obtained by cropping pictures between the hair line and the chin with the image manipulation software Gimp 2 (http://www.gimp.org). The face was given an oval shape by tracing an ellipse in a rectangle of $25 \times 35$ pixels. Extraneous background information 


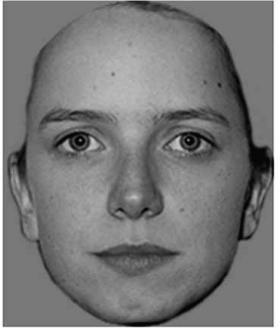

(a)

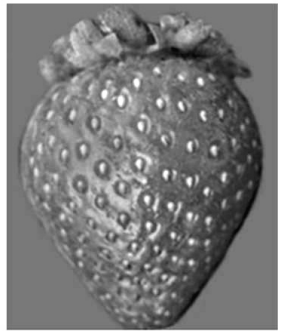

(b)
Figure 2. Examples of a facial stimulus (a) and of an object stimulus (b).

was concealed (see figure 2a). The object stimuli were constructed with the same parameters as those used for faces (see figure 2b). Contrast and luminance of each of these pictures were equalised by the experimenter. Each of the four objects and of the four faces was presented as CS to six different participants.

The arms of the cross that served as the stimulus for the length-judgment task were black and had 4 different sizes ranging between $3.6 \mathrm{deg}$ and $4.5 \mathrm{deg}$ (the two intermediate sizes being 3.9 and $4.1 \mathrm{deg}$ ). The mask was a square with a side of $8.9 \mathrm{deg}$ covering the area in which stimuli could appear. All these stimuli were presented on a medium-grey background (see figure 3).

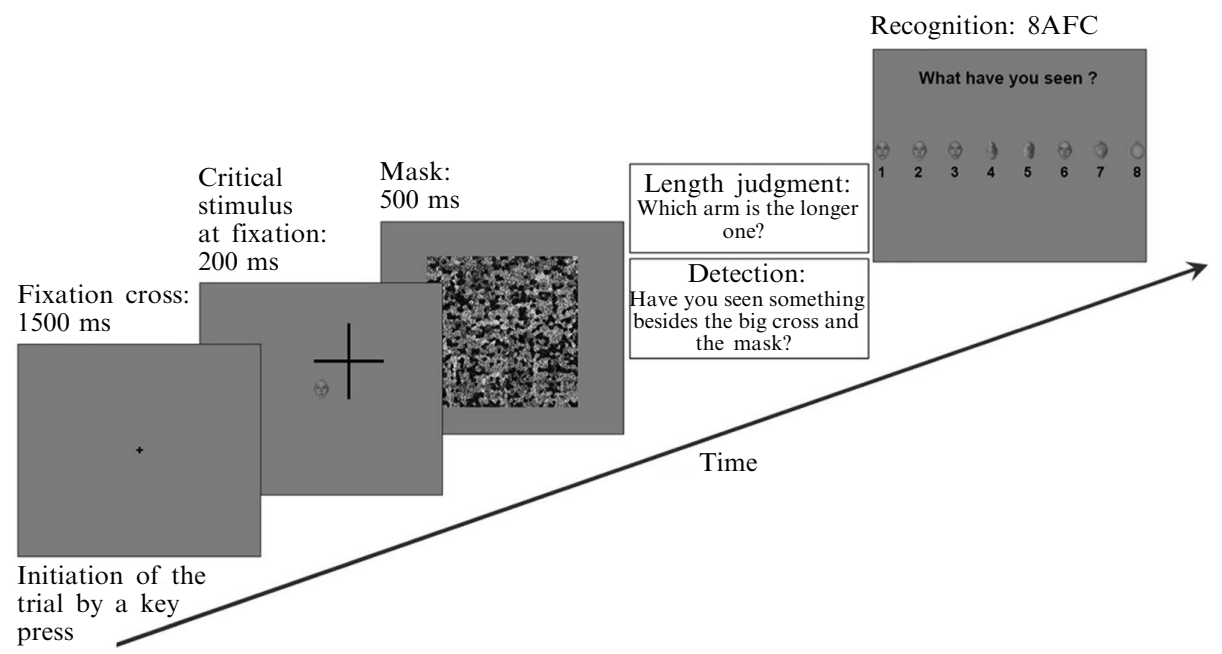

Figure 3. Time course of a critical trial in experiments 1, 2, and 3.

2.1.3 Procedure. Participants were tested individually in a dimly lit room on a personal computer. They viewed stimuli at a distance of $56 \mathrm{~cm}$ controlled by means of a chinrest. The stimuli were presented on a 17-inch CRT monitor with an $85 \mathrm{~Hz}$ refresh rate and the resolution of the screen was set to $1024 \times 768$ pixels. The presentation of the stimuli was controlled and responses were recorded with E-Prime 1.0 software (http:// www.pstnet.com/products/e-prime). Participants were instructed that they will perform a task on geometrical shapes. They were asked to stare at a centred fixation cross and to judge which arm of a larger cross presented very briefly was longer. Each trial was initiated by a key-press by the participant when he/she was ready. The fixation cross was presented for $1500 \mathrm{~ms}$. Then, a larger cross appeared for $200 \mathrm{~ms}$ randomly in one of the four quadrants on a $45^{\circ}$ diagonal from the fixation, the intersection of the arms being at $2.3 \mathrm{deg}$ from the fixation. The two arms of the cross were chosen randomly among the four possible sizes with the constraint that both of them could not have the same size. Participants indicated their decision with a key-press ('b' = vertical or 
' $n$ ' = horizontal). During critical and control trials, a CS appeared at fixation (ie at the location of the fixation cross) for $200 \mathrm{~ms}$ at the same time as the larger cross. Finally a mask was presented for $500 \mathrm{~ms}$.

Each participant was given 9 trials (see table 1). CS was presented during the fourth (inattentional), the eighth (divided attention), and the ninth (control) trial. The same CS was presented throughout the whole experiment to a given participant. After each critical trial, participants were asked if they had seen anything besides the large cross and the mask (referred to as the black-and-white spotted square) that was not present during previous trials. Regardless of their response, they had to choose what had just been presented in an $8 \mathrm{AFC}$ composed of the four faces and the four objects. These eight items randomly appeared in one of eight possible locations (see figure 3). In order to assess whether participants were prone to produce a false positive, the CS was not presented during the sixth (bias) trial. However, the participants were still questioned about the presence of something additional and they had to pick out what 'had been presented' in the 8AFC. Before the ninth (control) trial, participants were urged to concentrate on the centre of the screen and to stare at the fixation cross, no longer paying attention to the large cross. They were again asked if they had seen something additional and had to pick it out in the 8AFC.

Table 1. Illustration of the procedure used in experiments 1, 2, and 3.

\begin{tabular}{|c|c|c|c|c|c|c|c|c|}
\hline Trial 1 & Trial 2 & Trial 3 & $\begin{array}{l}\text { Trial } 4 \\
\text { (inattention) }\end{array}$ & Trial 5 & $\begin{array}{l}\text { Trial } 6 \\
\text { (bias) }\end{array}$ & Trial 7 & $\begin{array}{l}\text { Trial } 8 \\
\text { (divided } \\
\text { attention) }\end{array}$ & $\begin{array}{l}\text { Trial 9 } \\
\text { (control) }\end{array}$ \\
\hline \multirow[t]{2}{*}{$\begin{array}{l}\text { cross } \\
\text { alone }\end{array}$} & $\begin{array}{l}\text { cross } \\
\text { alone }\end{array}$ & $\begin{array}{l}\text { cross } \\
\text { alone }\end{array}$ & $\begin{array}{l}\text { cross }+ \\
\text { critical } \\
\text { stimulus }\end{array}$ & $\begin{array}{l}\text { cross } \\
\text { alone }\end{array}$ & $\begin{array}{l}\text { cross } \\
\text { alone }\end{array}$ & $\begin{array}{l}\text { cross } \\
\text { alone }\end{array}$ & $\begin{array}{l}\text { cross }+ \\
\text { critical } \\
\text { stimulus }\end{array}$ & $\begin{array}{l}\text { to-be-ignored } \\
\text { cross + critical } \\
\text { stimulus }\end{array}$ \\
\hline & & & $8 \mathrm{AFC}$ & & $8 \mathrm{AFC}$ & & $8 \mathrm{AFC}$ & $8 \mathrm{AFC}$ \\
\hline
\end{tabular}

\subsection{Results}

Since the present study was aimed at evaluating the ability of faces or other stimuli to capture attention when they appear unexpectedly in condition of inattention, we focused our analyses on the inattention trial (ie first critical trial) for all analyses reported hereafter. Moreover, in the 'detection rates' section, we examined the tendency of participants to report the presence of an additional item although nothing has actually been presented by means of the bias trial.

2.2.1 Length-judgment task. First, the performance on the primary task (ie line-length judgment) was analysed. The overall accuracy in this task was $66 \%$. A $\chi^{2}$ analysis showed that the accuracy did not differ significantly between the two groups in the inattention trial $\left(\chi_{1}^{2}=2.42, p=0.12\right)$. Moreover, the rates of correct responses in critical trials (trials 4 and 8 being pooled), $68 \%$, did not differ significantly from that in adjacent non-critical trials (trials $3,5,6$, and 7 being pooled) $\left(71 \%, \chi_{1}^{2}<1\right)$, which suggests that the presence of a CS did not significantly divert attention from the length-judgment task.

2.2.2 Detection rates. We compared the detection rates as a function of the type of CS presented (ie face versus object) during the first critical trial by means of a $\chi^{2}$ analysis. Faces $(58 \%)$ were significantly better detected than objects $(4 \%)\left(\chi_{1}^{2}=16.39\right.$, $p<0.001)$. In addition, the bias trial indicated that only two participants from the 'face' condition ( $8 \%$ ) and three from the 'object' condition (12.5\%) reported seeing something, although nothing had been presented. Complete results are presented in the left panel of figure $4 \mathrm{a}$. 


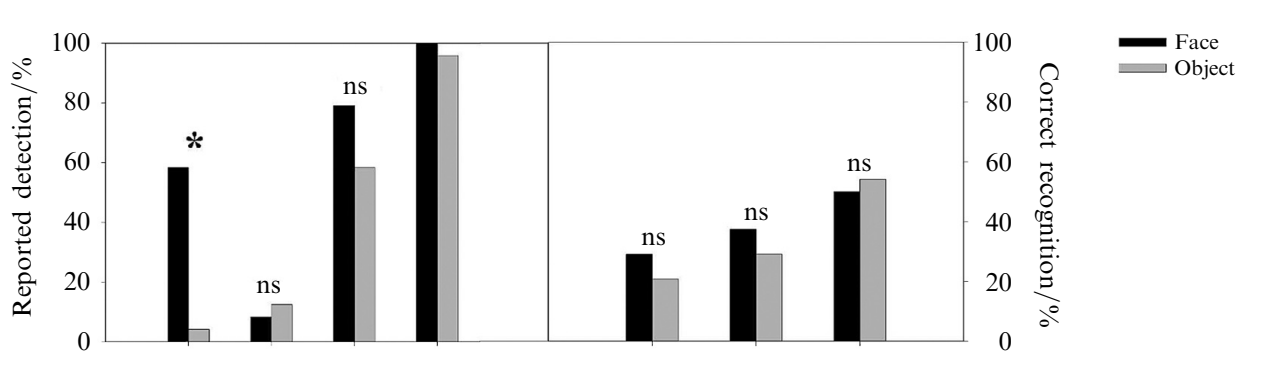

(a)

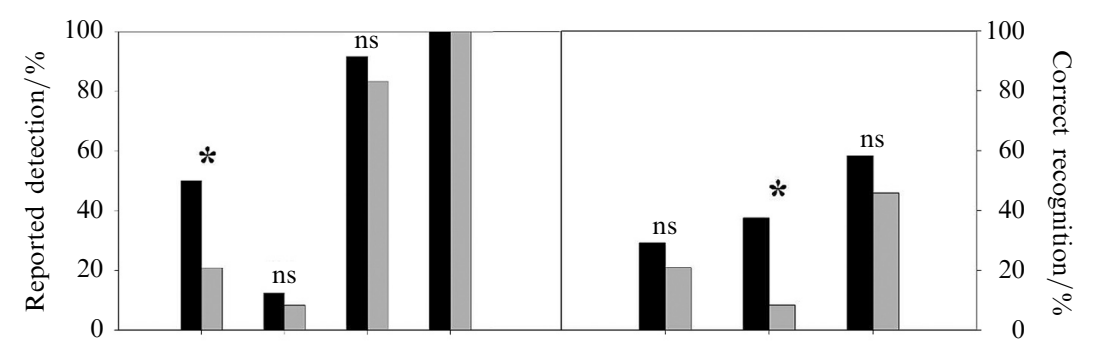

Upright face

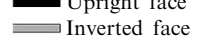

(b)

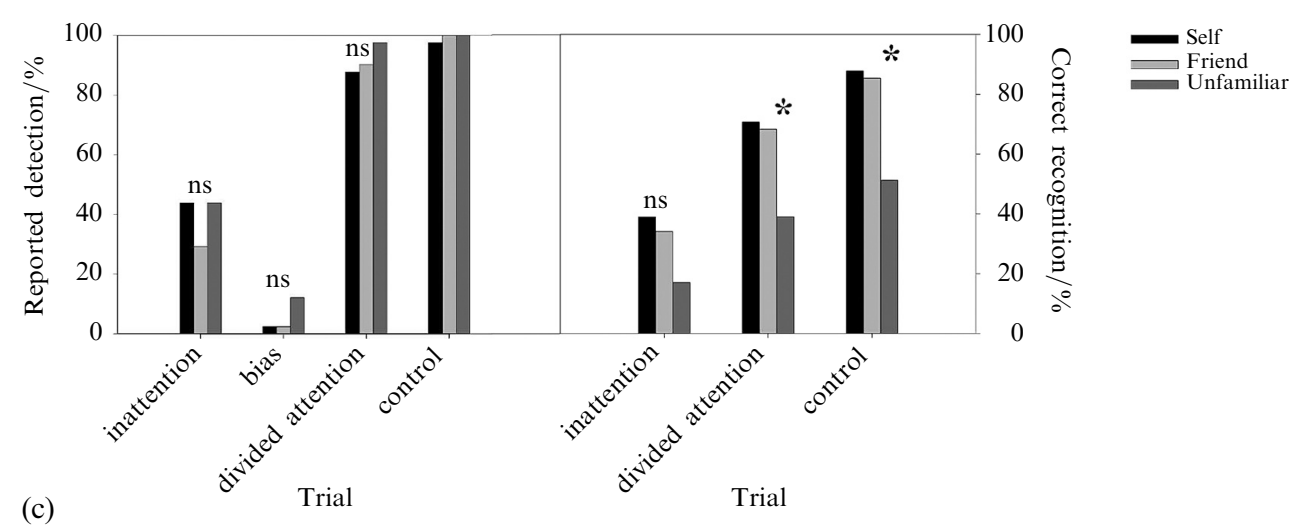

Figure 4. Left panels: Percentage of participants that reported having detected an additional stimulus as a function of the type of critical stimulus (CS) presented in critical and bias trials of (a) experiment 1, (b) experiment 2, and (c) experiment 3. Right panels: Percentage of correct recognition of the CS judged by $8 \mathrm{AFC}$. * Refers to a significant $p$ value $<0.05\left(\chi^{2}\right.$ analyses); $\mathrm{ns}$ refers to a non-significant $p$ value $>0.05$. Note that we did not conduct analyses on the detection rates of the control trial because performance was at ceiling. This control trial indicates that the CSs were readily visible in conditions of full attention.

2.2.3 Correct recognition rates. We compared the ability of participants to recognise the CS that has been presented in the inattention trial by means of another $\chi^{2}$ analysis. The correct recognition rates did not differ significantly between faces $(29 \%)$ and objects $(21 \%)\left(\chi_{1}^{2}<1\right)$. The results are presented in the right panel of figure $4 \mathrm{a}$. Finally, for the sake of exhaustiveness, results of analyses examining the recognition rates depending on whether the presence of the critical stimulus had been reported or not are presented in figure 5a. These analyses show no significant differences in the ability to recognise faces and objects, either when they had or had not been detected, and on any of the trials (inattention, divided attention, and control). 

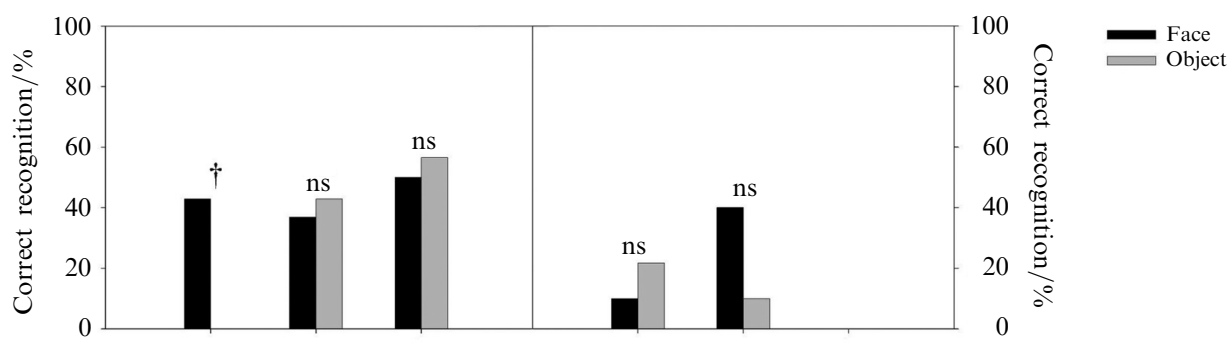

(a)
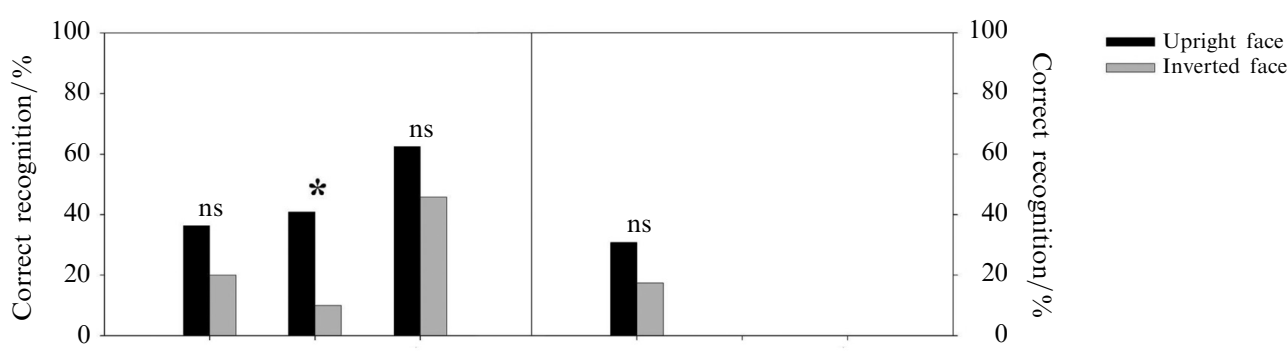

(b)
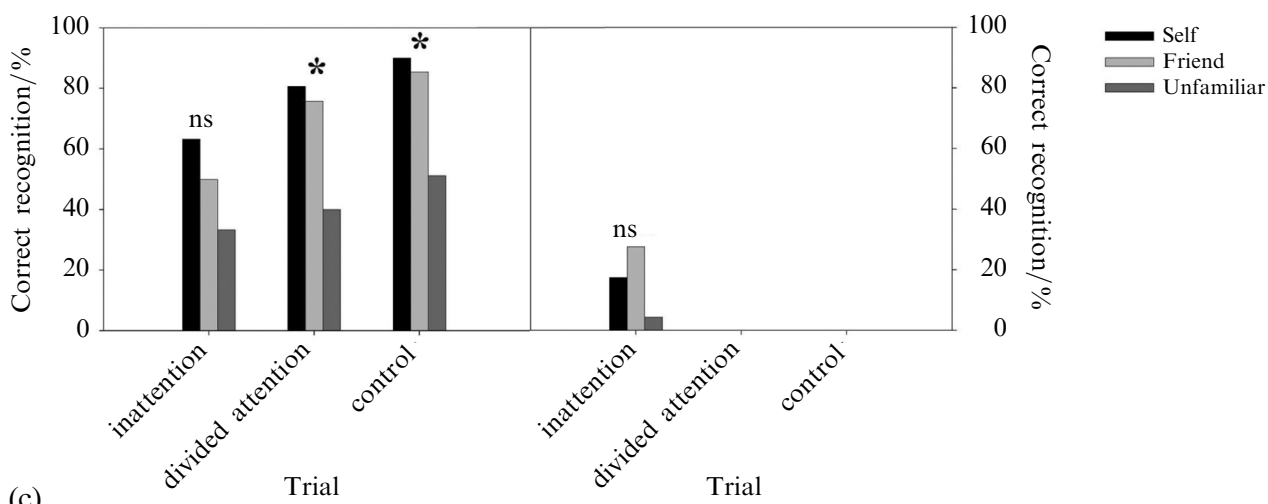

Figure 5. Percentage of correct recognition of the critical stimulus (CS) depending on whether its presence was reported (left panels) or not (right panels) in (a) experiment 1, (b) experiment 2, (c) experiment $3 . *$ refers to a significant $p$ value $<0.05$ ( $\chi^{2}$ analyses); ns refers to a non-significant $p$ value $>0.05$. $\dagger$ A statistical analysis was not possible here because of an insufficient number of observations in the 'object' condition. Note that the absence of correct recognition of undetected CSs on divided attention and control trials is due to detection rates (see the left panels of figure 4) that were at or near ceiling on those trials.

\subsection{Discussion}

Current results confirm previous findings, this time with realistic photographic stimuli, that faces are more resistant to IB than are other objects (Mack and Rock 1998). In general terms, this finding is consistent with the idea that faces are more likely to grab attention than other objects (Mack et al 2002; see also Ro et al 2001; Theeuwes and Van der Stigchel 2006; but see Palermo and Rhodes 2003). The present experiment, this time with realistic photographic stimuli, also shows an overall IB effect of $69 \%$. 
The present findings thus provide a confirmation of previous findings demonstrating the robustness of the IB effect (otherwise already demonstrated in more complex situations such as dynamic events; see Simons and Chabris 1999).

Although faces were better detected than objects during the inattention trial, recognition rates did not differ significantly between faces and objects. This shows that despite the fact that they are not easily identifiable, faces possess the ability to capture attention more than other categories of objects. This poor recognition performance is probably due to the difficulty of recognising a small novel face, presented unexpectedly while participants are engaged in another task, after a relatively short exposure (eg Bruce et al 2001). Crucially though, even when participants were not capable of recognising a face in such conditions, this face nevertheless had a strong power to attract attention and reduce IB rates dramatically.

The bias trial also indicated that few participants ( 5 of $48=10.4 \%$ ) were biased to report seeing something when nothing was actually presented. This suggests that those reporting seeing something in the first critical trial really did so because most participants were able to correctly report that nothing was presented. ${ }^{(1)}$

It cannot be excluded that the results of experiment 1 reflect some low-level differences between faces and other objects. For instance, because of their inner features, faces could be less homogenous than objects, rendering the former more readily detectable than the latter. To address this possibility, we conducted a second experiment in which we compared the detection of upright and inverted faces.

\section{Experiment 2}

The aim of this second experiment was thus to compare faces to another kind of CSs that have exactly the same low-level properties as faces (contrast, luminance, complexity, etc) but are not perceived as faces-inverted faces. Indeed, it has been suggested that inverted faces are not processed as faces but rather as objects (eg Valentine 1988). Hence, if a detection advantage for upright faces compared with inverted faces is obtained, it would confirm the view that the advantage is due to their meaning rather than to other lower-level characteristics.

(1) However, one could argue that participants were not in the same situation during the inattention trial and during the bias trial in which attention was in fact already divided owing to the earlier presentation of the first critical trial (we thank Ian Thornton for this suggestion). In order to answer this potential criticism, we recruited eighteen new participants to whom the first critical trial (inattention) was not presented. In this case, following three non-critical trials, the bias trial constituted the first trial after which participants were asked whether they had detected something additional. Results showed that five participants $(28 \%)$ reported seeing something although nothing had been presented. When asked to describe what they had seen before the 8AFC, not surprisingly three participants were not able to answer and just had a feeling of having seen something, one described a picture composed of points appearing after the big cross and before the mask, and one described a strange large image with plants. These two descriptions clearly fit the appearance of the mask and indicate that some participants may confuse the perception of the mask with that of an additional item. These participants probably noticed the mask because of the question, and confused it with something additional because they had not noticed it before. It is thus possible that the rates of false positives seemed higher when nothing was presented at first because participants did not know what there was to 'see', whereas when the bias trial was presented after a genuine critical trial people knew what could be presented and in consequence were more sure that they had not seen such a thing. This problem of false positives had already been addressed by Mack and Rock (1998). In a control experiment similar to that conducted here, they found that $25 \%$ of participants reported that there was something although nothing had been presented. Thus, according to Mack and Rock (1998), "to avoid appearing either dumb or blind, these subjects may have answered 'yes' to our question even though they actually had not seen anything else" (page 238). They concluded that the rate of IB is generally underestimated, since these cases are actually additional cases of IB. 


\subsection{Method}

3.1.1 Participants. We recruited forty-eight new participants (eleven men) from the University of Liège. They were randomly assigned to one of two experimental conditions: upright face $(n=24)$ or inverted face $(n=24)$ as CS. They were naive as to the purpose of the experiment and had normal or corrected-to-normal vision. They gave their informed consent prior to their inclusion in the experiment.

3.1.2 Stimuli and procedure. The stimuli and the procedure were exactly the same as in experiment 1, except that the four objects used in experiment 1 were replaced by the four unfamiliar faces that had been flipped vertically. Each of these eight CSs was presented throughout the whole experiment to six different participants. The 8AFC now comprised four upright faces and the same four inverted faces.

\subsection{Results}

3.2.1 Length-judgment task. The overall accuracy in the length-judgment task was 65\% which is similar to the $66 \%$ obtained in experiment $1 . \chi^{2}$ analyses showed that the accuracy did not differ significantly between the two groups in the inattention trial $\left(\chi_{1}^{2}=1.37, p=0.24\right)$. Moreover, the rates of correct responses in critical trials (trials 4 and 8 being pooled), 59\%, did not differ significantly from that in adjacent non-critical trials (trials $3,5,6$, and 7 being pooled), 66\% $\left(\chi_{1}^{2}=1.27, p=0.26\right)$.

3.2.2 Detection rates. As in experiment 1 upright faces resisted more to IB than other stimuli. Indeed, upright faces $(50 \%)$ were significantly better detected than inverted faces $(21 \%)\left(\chi_{1}^{2}=4.46, p=0.035\right)$. Moreover, the bias trial indicated that only three participants from the upright-face condition (12.5\%) and two from the inverted-face condition $(8 \%)$ reported the presence of an additional item when nothing had actually been presented. Complete results are shown in the left panel of figure $4 \mathrm{~b}$.

3.2.3 Recognition rates. The correct recognition rates did not differ significantly between upright faces $(29 \%)$ and inverted faces $(21 \%)$ after their first appearance $\left(\chi_{1}^{2}<1\right)$. The results are presented in the right panel of figure $4 \mathrm{~b}$. In addition, figure $5 \mathrm{~b}$ illustrates the recognition rates depending on whether the presence of the critical stimulus had been reported or not. Upright faces were not significantly better recognised than inverted faces, except in the divided attention trial after participants reported the detection of the CS.

\subsection{Discussion}

This second experiment confirms the findings of experiment 1 that faces are more resistant to IB than other stimuli. Here the stimuli in the comparison were the same faces, either upright or inverted. Since upright and inverted faces had exactly the same low-level properties, this detection advantage for upright faces is thus ascribable to their meaning. In the present experiment, the overall IB rates were $64.5 \%$ which is consistent with the IB effect found in experiment 1 and again confirms the robustness of the IB effect. In line with experiment 1, few participants ( 5 of $48=10.4 \%$ ) reported seeing something when nothing was actually presented during the bias trial.

Finally, as in experiment 1, upright faces benefited from a detection advantage without being better recognised than inverted faces after their first appearance.

In the preceding experiments we showed that an unfamiliar face grabs more attention than other stimuli. In the next experiment we examine whether familiarity and identity of a face influence its ability to capture attention. 


\section{Experiment 3}

In this experiment, the resistance to IB of three types of faces differing in identity and familiarity was compared. The CS was either an unfamiliar face (as in experiments 1 and 2) or a familiar face. In the latter case, the CS was either the participant's own face or the faces of a participant's friend.

\subsection{Method}

4.1.1 Participants. One hundred and forty-four participants (thirty-seven men) from the University of Liège participated in the experiment on a voluntary basis. Each of them had been photographed previously in the Cognitive Psychology Unit for their participation in the recognition of one's own and familiar-face experiment (each participant had taken part in these experiments with a friend/colleague so that each participant served as the familiar control for the other member of the pair). They were recruited by phone (on average 11 months after they had been photographed) and were asked to participate in a visual perception experiment about geometrical shapes that appeared to be completely unrelated. Participants were randomly assigned to one of three experimental conditions: own face $(n=48)$, friend's face $(n=49)$, and an unknown face $(n=47)$ as CS. They were naive as to the purpose of the experiment and had normal or corrected-to-normal vision. They gave their informed consent prior to their inclusion in the experiment.

4.1.2 Stimuli. Now, the CS was either a grey-scale picture of a familiar or of an unfamiliar face. These facial stimuli were constructed with the same parameters as those described above. When the CS was unfamiliar (unknown-face condition), it was chosen from a set of six unknown faces (three males and three females). When the CS was a familiar face, it was either the participant's own face (own-face condition) or the face of the participant's friend (friend's-face condition).

4.1.3 Procedure. The procedure was exactly the same as in experiments 1 and 2 except that the $8 \mathrm{AFC}$ was now tailored for each participant. The $8 \mathrm{AFC}$ was always composed of four faces of the same gender as the participant and of the same four objects as in experiment 1 . The four faces were two unknown faces and two familiar faces (the participant's own face and his/her friend's face). Hence, whatever the group the participant belonged to he/she had to choose between two familiar and two unfamiliar faces and could not make a default decision based on face familiarity if he/she had not really recognised it. The four objects served as filler items in the 8AFC and were never presented as CS during this experiment.

In addition, after the 9 trials, participants were presented with the four faces and were asked to identify them. To make sure that the possible differences obtained between the three groups of participants were due to the different familiarity of the CS they had been presented with, participants who were not able to identify one (either their own face or their friend's face) or both familiar faces were discarded.

\subsection{Results}

Twenty-one participants could not identify at least one of the two familiar faces during the final identification phase (seven participated in the own-face condition, eight in the friend's-face condition, and six in the unknown-face condition) and were discarded from further analyses (there were thus forty-one participants per condition). ${ }^{(2)}$

(2) Nonetheless, it should be mentioned here that most of these twenty-one participants recognised the stimuli as being their own faces and their friend's faces after we told them. Some of them admitted that they thought they had recognised the faces but did not dare to say so. Some of them were also a bit stunned, as they did not understand how the pictures of these faces could have ended up in the present experiment. 
4.2.1 Length-judgment task. The overall accuracy in the length-judgment task was $69 \%$, which is in line with the two previous experiments. $\chi^{2}$ analyses showed that the accuracy did not differ significantly between the three conditions in the inattention trial $\left(\chi_{2}^{2}=2.36, p>0.25\right)$. Performance in critical trials (trials 4 and 8 being pooled), $70 \%$, did not differ significantly from that in adjacent non-critical trials (trials $3,5,6$, and 7 being pooled), $75 \%\left(\chi_{1}^{2}=1.52, p=0.22\right)$.

4.2.2 Detection rates. Here we focused again on the inattention trial to examine whether the self-relevance and familiarity of faces can influence their resistance to IB. The null hypothesis that all three types of faces are equally resistant to IB could not be rejected since the facial identity did not significantly influence the detection rates (own face $=44 \%$, friend's face $=29 \%$, unknown face $=44 \% ; \chi_{2}^{2}=2.46, p>0.25$ ). However, to ensure that this numerical difference of $15 \%$ between the friend's-face condition and the two other conditions did not reflect a genuine effect that the global $\chi^{2}$ could not reveal, we performed two $2 \times 2$ analyses with the friend's-face condition tested against the two other conditions. These two analyses did not reveal significant differences between the friend's-face condition and the other conditions (both $\chi_{1}^{2}=1.89, p=0.17$ ).

At the bias trial, only one participant in the own-face condition (2.44\%), one in the friend's-face condition (2.44\%), and five in the unknown-face condition $(12.20 \%)$ reported seeing something when nothing additional was actually presented. Complete results are presented in the left panel of figure 4c.

4.2.3 Correct recognition rates. A $\chi^{2}$ analysis showed that the familiarity of the face tended to increase the recognition of the CS presented during the inattention trial (own face $=39 \%$, friend's face $=34 \%$, unknown face $=17 \%$ ). However, the difference between the three groups was not significant $\left(\chi_{2}^{2}=5.18,0.10>p>0.05\right)$. The right panel of figure $4 \mathrm{c}$ nonetheless shows that this advantage of both familiar faces over the unknown face was significant in the divided-attention trial and in the control trial. Figure $5 \mathrm{c}$ illustrates the recognition rates conditioned on whether the presence of the critical stimulus had been reported or not. In the inattention trial, the familiarity of the faces did not significantly enhance their recognition either when they had been detected or not. By contrast, familiarity significantly affected recognition rates on subsequent divided-attention and control trials for detected items.

4.2.4 Bias trial. We examined the participants' choices after the bias trial to evaluate whether they were biased to think that the experiment dealt with their own face and therefore that their own faces had been presented. Overall (ie irrespective of participants reporting the presence of an additional item or not), twelve participants $(9.76 \%)$ chose their own face when nothing had actually been presented: two $(4.9 \%)$ from the own-face condition, seven (17.1\%) from the friend's-face condition, and three $(7.3 \%)$ from the unknown-face condition. In addition, binomial tests (confidence interval thresholded at $\alpha=0.05$ ) were used to assess whether these rates differed from what had been expected by chance (ie 12.5\%). Participants from the friend's-face and unknownface conditions chose their own face at random and participants from the own-face condition chose their own face less often than expected by chance when nothing had been presented.

Among participants who reported having seen something in the bias trial (ie seven participants, see above), none chose the own face in the own-face condition, one did so in the friend's-face condition and none did it in the unknown-face condition.

\subsection{Discussion}

Unexpectedly, the results of this third experiment do not suggest any significant influence of self-relevance or of face familiarity on detection rates since all faces grabbed 
attention similarly. A first explanation for this null effect is that the combination of two properties likely to grab attention (ie facial aspect and self-relevance) does not increase the resistance of own face to IB. Indeed, it seems that the detection rates reported when own face was presented (ie 44\%) were similar to those found for unfamiliar faces across our three experiments (44\% in the present experiment, $58 \%$ in experiment 1 , and $50 \%$ in experiment 2 - thus $51 \%$ on average).

However, our results indicated a non-significant numerical difference of $15 \%$ between the reported detection rates for a friend's face (29\%) and the two other types of faces (44\% for both). Therefore, an alternative explanation for this null effect might simply be a lack of statistical power. Indeed. IB experiments require a large number of subjects in order to reach good power and, because participants were unexpectedly presented with their own faces and faces of their friends, we were limited by the number of participants we could recruit. Nevertheless, while there might be a power issue regarding a potential difference between friend's face and other faces, there seems to be no power issue regarding the comparison between own face $(44 \%)$ and unknown faces (also 44\%). So, even if the difference between friend's face and other faces was significant, this would not mean that own face particularly attracts attention but rather that friend's face is less prone to attract attention than other faces.

Nonetheless, there are two points that suggest that the non-significant difference between the friend's-face condition and the two other conditions does not reflect a genuine difference in the attention-grabbing capacity of such a highly familiar face. First, such a decrease in detection for a friend's face in comparison with a less familiar face (unknown face) is quite unexpected. We would have expected a linear effect where the attention-grabbing capacities decreased as the familiarity decreased (ie own face $>$ friend's face $>$ unfamiliar face - see eg Buttle and Raymond 2003). Second, a more thorough analysis of recognition data showed that a friend's face, unlike the others, was relatively well recognised when participants did not report that they had seen something additional after the first critical trial (see the right panel of figure 5c). Indeed, in that situation $28 \%$ of participants accurately recognised their friend's faces, against $17 \%$ that recognised their own faces and $4 \%$ that recognised unfamiliar faces. Binomial tests indicated that the recognition of undetected CS was at random level for own face [critical interval $(\mathrm{CI})=3 \%$ to $28 \%$ ] and for an unknown face (CI $=3 \%$ to $28 \%$ ) but significantly higher than random level for a friend's face $(\mathrm{CI}=4 \% \text { to } 27 \%)^{(3)}$ In other words, although they reported that they did not detect the presence of an additional item, participants from the friend's-face condition picked out their friend's face in the 8 AFC more often than expected by chance. This could indicate that some participants who detected their friend's faces did not report it (eg because they did not remember that they had previously participated in an experiment in which they were paired with this particular person, found it 'weird' to perceive such a picture, and were unsure of their perception) but nonetheless recognised it in the 8AFC. This explanation is plausible if one imagines the situation of the participant. $\mathrm{He} / \mathrm{she}$, in most cases, is tested by an experimenter he/she does not know, on average 11 months after having been photographed to participate in another familiar-faces experiment with his/her friend. Thus, it seems quite unlikely that this experimenter knows the identity of his/her friends and in addition have their pictures (see also footnote 2)! In this situation, they had some reasons to be reluctant to report such a perception.

At the bias trial, as in the two other experiments, ${ }^{(4)}$ there were only a few false positives since only seven participants out of one hundred and twenty-three $(5.70 \%)$

(3) Even though $\chi^{2}$ analysis showed no significant effect of the type of face, as reported in the right panel of figure $5 \mathrm{c}$.

(4) The reported detection rate in the present experiment was not significantly different from that obtained in experiment $1\left(\chi_{1}^{2}=1.18, p=0.28\right)$; or in experiment $2\left(\chi_{1}^{2}=1.18, p=0.28\right)$. 
reported seeing something when nothing was actually presented. The bias trial also allowed us to verify whether participants were biased to think that their own face had been presented because of their previous participation in an own-face experiment. Apparently, this was not the case, since participants were less than $10 \%$ likely to choose their own face when nothing was presented (ie after the bias trial). Moreover, after the bias trial, in all three conditions the choice of own face was not higher than at random level and actually, in the own-face condition, the choice of own face was even lower than expected by chance.

Finally, recognition rates indicated that familiar faces tended to be better recognised than unfamiliar faces even if the former were not more resistant to IB than the latter (see figure $4 \mathrm{c}$ ).

\section{General discussion}

Previous studies with the IB paradigm have shown that some categories of important and meaningful stimuli, such as faces or self-referential materials (eg the participant's own name), particularly capture attention and resist IB more than stimuli of a lesser importance (Mack and Rock 1998). Here, we aimed to investigate the resistance of such important stimuli to IB with realistic facial stimuli, and to investigate further the role of familiarity and self-relevance in the IB phenomenon.

\subsection{Detection rates and resistance of facial stimuli to $I B$}

Experiment 1 showed that faces are significantly better detected than pictures of common objects (58\% versus $4 \%$ of detection) under condition of inattention, confirming, with more realistic stimuli, previous findings about the special attentional properties of faces (Mack and Rock 1998; Ro et al 2001; Theeuwes and Van der Stigchel 2006; but see Palermo and Rhodes 2003). In addition, the results of experiment 2 showed that upright faces are also better detected than inverted faces (50\% versus $20 \%$ of detection), ruling out any alternative explanation in terms of potential low-level inherent differences between faces and objects. Finally, the results of experiment 3 showed no significant difference between the detection rates of one's own face $(44 \%)$, a friend's face $(29 \%)$, and an unknown face (44\%). In addition, one's own face was clearly detected in the same range as an unknown face in all three experiments. In other words, even though one's own face combines two properties likely to grab attention, it does not resist more to IB than another face. There are two possible interpretations of this result:

(i) Self-referential stimuli have no special abilities to capture attention. The first possibility is that self-relevance itself does not particularly capture attention in conditions of inattention (as shown in experiment 3) and that a face is sufficiently important and meaningful to capture attention whatever its identity (as shown in experiments 1 and 2). This would be in agreement with previous findings showing that self-referential stimuli do not automatically capture attention (Bundesen et al 1997; Gronau et al 2003; Harris et al 2004; Harris and Pashler 2004; Kawahara and Yamada 2004; Laarni et al 2000). The conditions in which self-referential stimuli have been shown to elicit attentional bias by comparison with other stimuli were as follows: when they were presented as distractors but their processing was somehow related with the ongoing task (Brédart et al 2006; Gronau et al 2003; Kawahara and Yamada 2004), when-if unrelated to the ongoing task - they were presented centrally (ie within the observer's focus of attention-see Devue and Brédart 2008; Gronau et al 2003) and when enough attentional resources were available for their processing (Harris and Pashler 2004). Therefore, the current study indicates another situation in which self-referential stimuli might not be particularly prone to capture attention: when they are unexpectedly and briefly presented at the centre of the visual field while observers are engaged in another unrelated and demanding task. 
(ii) Need of attention to process identity. The second interpretation of our results could be that attentional capture is indeed determined by meaning and significance (see Mack and Rock 1998), but by a 'rough' meaning, at a processing stage where precise semantic details and fine sub-categorisation are not yet available (ie a 'not that late' selection of attention - see eg Lachter et al 2004 for a similar view). Indeed, previous models of face recognition (eg Bruce and Young 1986) suggest that faces may be processed at different levels of specificity, from a general level (ie face versus non-face decision) to a very specific level (ie determining the identity of a face). For faces to capture attention outside our direct attentional focus, perceptual processes must exist that automatically scan and analyse the visual field for face stimuli. Because faces capture attention, one has to assume that faces are discriminated by some 'pre-attentive' or unconscious processing (Theeuwes and Van der Stigchel 2006). Current findings suggest that the discrimination between a face and a non-face configuration could be based on this early pre-attentive processing (that could take place in the occipito-temporal cortex - see Devue et al 2007). However, in order to discern the identity of the face, a second 'attentive' processing stage (possibly originating in the fusiform gyrus - see Devue et al 2007) may be necessary. This could explain why faces are more likely to capture attention than other stimuli (see experiments 1 and 2), and faces with different identities and levels of familiarity do not differ in their attention-grabbing capabilities (see experiment 3). It would be interesting to address further the generalisability of our finding to other types of facial classification (eg gender, gaze direction, race, or emotional expression) with realistic photographic stimuli.

Further work is necessary to determine the exact reason for the present null effect. One might ask, however, why some other researchers found that self-referential stimuli capture or attract attention (eg Brédart et al 2006; Mack et al 2002; Mack and Rock 1998; Moray 1959; Shapiro et al 1997; Wolford and Morrison 1980) if this null effect reflects a genuine absence of difference in the capacity of different faces to resist IB. We propose a 'retention hypothesis' to explain this discrepancy. We suggest that terms such as attentional 'capture', 'attraction', or 'draw' might have been used inadequately.

It could be that in studies showing 'self effects' self-referential stimuli did not capture attention but actually caused a difficulty to disengage attention because the paradigms used allowed the participants to attend to those stimuli (eg if the self-referential stimuli were located within the observer's focus of attention-see Devue and Brédart 2008; Shapiro et al 1997; Wolford and Morrison 1980; or if task demands allowed attentional shifts towards peripheral self-referential stimuli-see Brédart et al 2006). In those studies 'self-effects' were either reflected in an increase or in a decrease in performance. On the one hand, self-referential stimuli could have an advantage (enhanced reportability - see eg Shapiro et al 1997; Wolford and Morrison 1980) over other stimuli. This advantage may be due to self-referential stimuli being processed more efficiently than less-familiar stimuli once they are located in the observer's focus of attention (see eg Bundesen et al 1997). This assumption is supported by our recognition data: once a face had attracted attention (because of its facial properties per se) and this face happened to be a familiar face, it was better recognised than a novel face. On the other hand, self-referential stimuli could also interfere with other tasks (ie an increase of reaction times or of error rates - see eg Devue and Brédart 2008; Harris and Pashler 2004; Wolford and Morrison 1980). This interference could be explained by a difficulty to disengage attention from those stimuli once they are attended by the observer. In other words, it is possible that, although the self-face may not capture attention, it nonetheless retains attention more than other faces once attention is focused on it (see Fox et al 2001 for a similar claim with fear-related stimuli). A recent eye-movement study (Devue et al 2009) supports this assumption by showing that one's own face does not receive a saccade faster in a visual-search task but is fixated 
longer than unfamiliar faces. Therefore, the absence of specific 'self-effects' in other studies could be accounted for by a difficulty to attend to these stimuli, resulting in an absence of attentional retention (eg if self-referential stimuli were located outside the focus of attention and their presentation was irrelevant for the task at hand-see Laarni et al 2000), rather than by a lack of attention capture.

\subsection{Recognition rates}

In our first two experiments faces were not better recognised than common objects (experiment 1) or than inverted faces (experiment 2) in the inattention trial, although faces were more readily detected than other stimuli. This is in line with our hypothesis formulated above that the visual system can detect facial structures pre-attentively but that further processing is necessary to access other information such as identity. Recognising a novel face after a short exposure seems quite difficult (eg Bruce et al 2001).

In experiment 3 , familiar faces were in general better recognised than unknown faces (after several presentations of the CS) even though all three faces similarly attracted attention. These recognition results are consistent with the two hypotheses formulated above to explain our detection results. Indeed, the recognition performance suggests that, once attention was on a face, the processing of familiar faces was facilitated in comparison with that of a face met for the first time. This advantage in the recognition of familiar faces has been shown previously (see for instance Bruce et al 2001) and is probably due to the fact that the robust representation built after an extensive experience with faces of highly familiar persons facilitates their processing (Tong and Nakayama 1999).

Note that the fact that the same CS was presented to a given participant throughout the experiment might have facilitated its recognition trials 8 and 9. However, after having seen the 8AFC following the first presentation of the CSs (as well as after the bias trial), participants knew exactly which stimuli could possibly be presented. This exposure to all the possible CSs probably also made the detection and the recognition of the CSs easier on trials 8 and 9 but this would have held if we had presented different CSs on trials 8 and 9.

\subsection{False positives and participants' bias}

In our three experiments, the bias trial (ie the sixth trial without CS) showed that false positives were quite unlikely (ie around or less than 10\%). This indicates that participants who reported the presence of the CS after the inattention trial did so accurately since they were capable of judging that nothing had been presented in the following bias trial.

Because it was presented after the inattention trial, the bias trial did not constitute a perfect measure of the occurrences of false positives. However, it was impossible to check the occurrence of false positives before the first critical inattention trial since an observer can be only once in the condition of inattention in the IB paradigm. Nonetheless, in the specific context of our third experiment, this trial allowed us to make sure that participants were not biased to think that the experiment dealt with their own faces. The results indicated that participants did not tend to infer that their own faces had been presented and that the responses they gave on the other trials were quite trustworthy.

\subsection{Conclusion}

To summarise, our results showed that pictures of unknown faces were better detected than pictures of common objects or inverted faces. Moreover, the self-relevance and the familiarity of a face did not affect its resistance to IB. However, once a familiar face was attended, its processing was facilitated by comparison with that of a novel face. 
This study gives rise to novel hypotheses that should be investigated. The first possibility is that self-referential materials have no special attention-grabbing capacities, at least in the conditions defined by the IB paradigm. The second possibility is that the precise meaning of a facial stimulus is not yet available at the instant attention is captured, and that attention is necessary to process identity.

Acknowledgments. This research was supported by a grant from the Belgian Fonds de la Recherche Fondamentale Collective (Grant 8.4506.05-2.4539.05) to SB. CD is a postdoctoral researcher at the National Fund for Scientific Research (Belgium).

\section{References}

Brédart S, Delchambre M, Laureys S, 2006 "One's own face is hard to ignore" Quarterly Journal of Experimental Psychology $\mathbf{5 9} 46-52$

Bruce V, 1988 Recognizing Faces (Hillsdale, NJ: Lawrence Erlbaum Associates)

Bruce V, Henderson Z, Newman C, Burton M, 2001 "Matching identities of familiar and unfamiliar faces caught on CCTV images" Journal of Experimental Psychology: Applied 7 207-218

Bruce V, Young A, 1986 “Understanding face recognition” British Journal of Psychology 77 305- 327

Bundesen C, Kyllingsbaek S, Houmann K J, Jensen R M, 1997 "Is visual attention automatically attracted by one's own name?" Perception \& Psychophysics $59714-720$

Buttle H, Raymond J E, 2003 "High familiarity enhances visual change detection for face stimuli" Perception \& Psychophysics $\mathbf{6 5} 1296-1306$

David E, Laloyaux C, Devue C, Cleeremans A, 2006 "Change blindness to gradual changes in facial expressions" Psychologica Belgica $46253-268$

Deutsch J, Deutsch D, 1963 "Attention: Some theoretical considerations" Psychological Review 87 $272-300$

Devue C, Brédart S, 2008 "Attention to self-referential stimuli: Can I ignore my own face?" Acta Psychologica $128290-297$

Devue C, Collette F, Balteau E, Degueldre C, Luxen A, Maquet P, Brédart S, 2007 "Here I am: The cortical correlates of visual self-recognition" Brain Research 1143 169-182

Devue C, Van der Stigchel S, Brédart S, Theeuwes J, 2009 "You do not find your own face faster; you just look at it longer" Cognition 111 114-122

Fox E, Russo R, Bowles R, Dutton K, 2001 "Do threatening stimuli draw or hold visual attention in sub-clinical anxiety?" Journal of Experimental Psychology: General 130681 - 700

Gronau N, Cohen A, Ben-Shakhar G, 2003 "Dissociations of personally-significant and taskrelevant distractors inside and outside the focus of attention: A combined behavioural and psychophysiological study" Journal of Experimental Psychology: General $132512-529$

Harris C R, Pashler H E, 2004 "Attention and the processing of emotional words and names: Not so special after all" Psychological Science $15171-178$

Harris C R, Pashler H E, Coburn N, 2004 "Moray revisited: High-priority affective stimuli and visual search" Quarterly Journal of Experimental Psychology 57 1-31

Kawahara J, Yamada Y, 2004 "Does one's name attract visual attention?" Visual Cognition 11 $997-1017$

Laarni J, Koljonen M, Kuistio A M, Kyröläinen S, Lempiäinen J, Lepistö T, 2000 "Images of a familiar face do not capture attention under conditions of inattention" Perceptual and Motor Skills $901216-1218$

Lachter J. Forster K I, Ruthruff E, 2004 "Forty-five years after Broadbent (1958): Still no identification without attention" Psychological Review $111880-913$

Mack A, Pappas Z, Silverman M, Gay R, 2002 "What we see: Inattention and the capture of attention by meaning" Consciousness and Cognition $11488-506$

Mack A, Rock I, 1998 Inattentional Blindness (Cambridge, MA: MIT Press)

Moray N, 1959 "Attention and dichotic listening: Affective cues and the influence of instructions" Quarterly Journal of Experimental Psychology $1156-60$

Ninomiya H, Onitsuka T, Chen C H, Sato E, Tashiro N, 1998 "P300 in response to the subject's own face" Psychiatry and Clinical Neurosciences $52519-522$

Palermo R, Rhodes G, 2003 "Change detection in the flicker paradigm: Do faces have an advantage?" Visual Cognition $10683-713$

Perrin F, Schnakers C, Schabus M, Degueldre C, Goldman S, Brédart S, Faymonville M E, Lamy M, Moonen G, Luxen A, Maquet P, Laureys S, 2006 "Brain response to one's own name in vegetative state, minimally conscious state, and locked-in syndrome" Archives of Neurology 63 $562-569$ 
Ro T, Russell C, Lavie N, 2001 "Changing faces: A detection advantage in the flicker paradigm" Psychological Science $1294-99$

Shapiro K L, Caldwell J, Sorensen R E, 1997 "Personal names and the attentional blink: A visual "cocktail party" effect" Journal of Experimental Psychology: Human Perception and Performance $23504-514$

Simons D J, Chabris C F, 1999 "Gorillas in our midst: Sustained inattentional blindness for dynamic events" Perception 281059 - 1074

Theeuwes J, Van der Stigchel S, 2006 "Faces capture attention: Evidence from inhibition of return" Visual Cognition 13 657-665

Tong F, Nakayama K, 1999 "Robust representations for faces: Evidence from visual search" Journal of Experimental Psychology: Human Perception and Performance 25 1016-1035

Valentine T, 1988 "Upside-down faces: A review of the effect of inversion upon face recognition" British Journal of Psychology $79471-491$

Vuilleumier P, Schwartz S, 2001 "Emotional facial expressions capture attention" Neurology 56 $153-158$

Wolford G, Morrison F, 1980 "Processing of unattended visual information" Memory \& Cognition $8521-527$ 


\section{PERTEPTION}

VOLUME 382009

www.perceptionweb.com

Conditions of use. This article may be downloaded from the Perception website for personal research by members of subscribing organisations. Authors are entitled to distribute their own article (in printed form or by e-mail) to up to 50 people. This PDF may not be placed on any website (or other online distribution system) without permission of the publisher. 